\title{
ELEVATED SERUM C-REACTIVE PROTEIN LEVEL IS NOT ASSOCIATED WITH SERUM NITRIC OXIDE IN PATIENTS WITH POSTTRAUMATIC STRESS DISORDER
}

\author{
Almir Fajkić ${ }^{1}$, Miralem Musić ${ }^{1}$, Alma Džubur Kulenović ${ }^{2}$, Asija Začiragić ${ }^{3}$, Nesina Avdagić ${ }^{3}$, \\ Nermina Babić ${ }^{3}$, Orhan Lepara ${ }^{3}$, Esad Pepić ${ }^{1}$, Amela Dervišević ${ }^{3} \&$ Jasminko Huskić $^{3}$ \\ ${ }^{l}$ Department of Pathophysiology, Medical Faculty, University of Sarajevo, Sarajevo, Bosnia and Herzegovina \\ ${ }^{2}$ Psychiatric Clinic, University Clinical Center Sarajevo, Sarajevo, Bosnia and Herzegovina \\ ${ }^{3}$ Department of Human Physiology, Medical Faculty, University of Sarajevo, Sarajevo, Bosnia and Herzegovina
}

received: 20.4.2017;

revised: 30.8.2017;

accepted: 20.9.2017

\begin{abstract}
SUMMARY
Background: The aim of the present study was to evaluate serum nitric oxide (NO) and C reactive protein (CRP) concentration in veterans with and without PTSD. Furthermore, we aimed to assess whether there is a correlation between serum NO and CRP concentrations in tested groups.

Subjects and methods: Cross-sectional study included 90 male individuals, with and without experience of direct war combat, divided into three equal groups (n=30): group 1-included war veterans with PTSD, group 2 - included war veterans without PTSD, and control group - 30 apparently healthy volunteers, without experience of direct war combat. The diagnosis of PTSD was assessed according to the guidelines in the $10^{\text {th }}$ revision of the International Classification of Diseases (ICD-10). High-sensitivity CRP was determined by immunonephelometry. The serum NO level was determined by classic colorimetrical Griess reaction.

Results: Serum CRP concentration in veterans with $(3.54 \pm 1.19 \mathrm{mg} / \mathrm{L})$ and without PTSD $(3.24 \pm 2.04 \mathrm{mg} / \mathrm{L})$, was significantly higher $(p<0.05)$ compared to control group $(1.26 \pm 1.06 \mathrm{mg} / \mathrm{L})$. Serum NO concentration in veterans with $(7.64 \pm 4.43 \mu \mathrm{mol} / \mathrm{L})$ and without PTSD $(7.12 \pm 2.60 \mu \mathrm{mol} / \mathrm{L})$ was significantly lower $(p<0.05)$ compared to control group $(11.26 \pm 7.01 \mu \mathrm{mol} / \mathrm{L})$. Statistically significant correlation between serum NO and CRP concentration was determined in veterans without PTSD $(r=-0.473 ; p<0.01)$. No correlation was observed between serum NO and CRP concentration in veterans with PTSD $(r=0.118 ; p=0.534)$ and in control group $(r=-0.067 ; p=0.727)$.

Conclusion: The present study has showed significant increase of serum CRP and significant decrease of serum NO concentrations in veterans with and without PTSD. Furthermore, statistically significant negative correlation between serum NO and CRP concentration was determined only in veterans without PTSD. Obtained results indicate that the complex mechanism of the pathogenesis of PTSD requires further research.
\end{abstract}

Key words: C-Reactive Protein - Nitric oxide - Posttraumatic Stress disorder - Veterans

\section{INTRODUCTION}

Posttraumatic stress disorder (PTSD) is a mental disorder that compromises balance in organism by affecting fundamental spheres of human functioning: psychological, physiological and social-interpersonal. PTSD has been long treated as exclusively psychiatric disorder. Recently numerous studies tried to establish role of complex neuroendocrine, immunological and inflammatory processes on molecular level, which are involved in PTSD development with consequences on whole organism (Yehuda 2002, Wong 2002, Altemus et al. 2006). Special emphasis is given to discovery of signal molecules, mediators and transmitters that are likely involved in PTSD pathogenesis, and their interrelations with the purpose of unraveling mechanism of PTSD development.

Nitric oxide (NO) is free radical gaseous molecule, easily dissolvable in lipids, which is present in all cells of human body. NO is synthesized enzymatically from amino acid L-arginine by three distinct isoforms of the enzyme, nitric oxide synthase (NOS). Two of these isoform are expressed in constitutive manner, predomi- nantly in the vascular endothelium (eNOS) and in the nervous system (nNOS). Under normal physiological condition these isoforms of NOS generate low, transient levels of NO in response to increases in calcium concentrations. These low levels of NO contribute to blood pressure regulation, platelet aggregation and adhesion, gastrointestinal motility and neurotransmission (Bredt 1999, Roberts et al. 2008, Guix et al. 2005). However, third isoform inducible NOS (iNOS) has special role. When it is induced by endotoxine and/or cytokines, it generates high sustained levels of NO. In certain conditions, NO would produce peroxynitrite contributing to cell cytotoxicity and tissue damage and up-regulation of the inflammatory response. In fact an excess of NO is involved in pathophysiology of numerous diseases, including cardiovascular and metabolic diseases (Omar et al. 2016), kidney disease (Orida \& Lai 2000), inflammatory bowel disease (Avdagić et al. 2013), neurological diseases (Ibragic et al. 2012), many tumors (Vannini et al. 2015) etc.

Studies have shown pivotal role of NO in physiological pathways of neurotransmission, immunological defense and homeostasis. It has been postulated that 
excess in NO production may have important role in development of cerebral ischemia, Alzheimer's disease, epilepsy and multiple sclerosis (Lucas et al. 2006). Decrease in NO production has been related to hypertension, angina pectoris and impotency (Moncada et al. 1991). Studies so far have been able to elucidate roles of NO in different physiological and pathopysiological conditions. However, data regarding importance of NO in PTSD pathogenesis are scarce and insufficient.

C-reactive protein (CRP) is sensitive systemic marker of inflammation, infection, and tissue damage. In vivo and in vitro studies have reported that CRP itself has pro-inflammatory and anti-inflammatory characteristics. Pro-inflammatory features of CRP are reflected in its ability to activate complement system, induce proinflammatory cytokines, and in inhibition of eNOS synthesis (Zaciragic et al. 2013). Anti-inflammatory features of CRP are less known. They are mainly reflected in its ability to increase release of anti-inflammatory cytokines such as IL-10, as well as, in its ability to stimulate expression of receptor IL-1 antagonists and in its ability to inhibit release of INF- $\gamma$ (McDade 2012). The role of inflammation in the pathogenesis of PTSD is far from being fully understood and studies that evaluated concentration of CRP in PTSD patients have given inconclusive and discordant results (Spitzer et al. 2010, Von Känel et al. 2007).

The aim of this study was to evaluate serum NO and CRP concentration in veterans with and without PTSD and compare them with values in the control group of healthy subjects. Furthermore, we aimed to assess whether there is a correlation between serum NO and CRP concentrations in tested groups. The hypothesis of the present study was that serum NO and CRP concentrations are different and that there is a correlation between NO and CRP in tested groups.

\section{SUBJECTS AND METHODS}

\section{Subjects}

Cross-sectional study, conducted at Clinic for Psychiatry Clinical Center University of Sarajevo (CCUS), included 90 Bosnian male individuals with and without experience of direct war combat. Subjects were divided into three groups: Experimental group 1included 30 veterans of male gender with experience of direct war combat, average age of $48.60 \pm 6.32$ years, with symptoms that qualify for PTSD diagnosis. Experimental Group 2 - included 30 veterans of male gender with experience of direct war combat, average age of $49.70 \pm 4.72$ years, without symptoms that qualify for PTSD diagnosis. Control group of subjects consisted of 30 apparently healthy volunteers, average age of $49.66 \pm 6.32$ years, without experience of direct war combat and without symptoms that qualify for PTSD diagnosis.
The participants from the PTSD group were recruited as a consecutive sample from the pool of patients who received out-patient treatment for PTSD in the Unit for Trauma-related Disorders at the Department of Psychiatry of the University Clinical Center Sarajevo. Participants from the non-PTSD and control group were recruited from the community, using the snowball method (Bloor et al. 1991).

In order to increase the validity of the results, we have defined the inclusion and exclusion criteria in a way that would enable better homogenization of groups of participants in most factors affecting the serum concentration of CRP and NO. Inclusion criteria were that all participants were male, aged between 40 and 60 , non-smokers, non-alcohol drinkers and did not take any prescription or non-prescription drugs that affected serum levels of CRP and NO or suffered from any somatic condition that affected these levels (acute and chronic inflammatory conditions, cancers, diabetes mellitus, hyperthyroidism, hypothyroidism, psoriasis, cerebrovascular insult, vascular dementia).

Detailed history was taken from all individuals included in the study, and objective physical and neuropsychiatric examination was done by trained specialist. Subjects from all three groups were tested for presence of PTSD according to the guidelines in the $10^{\text {th }}$ revision of the International Classification of Diseases (ICD-10) (WHO 2011). This study was conducted in according with the Helsinki Declaration and was approved by the Local Ethics Committee. With the informed consent of the subjects in the survey, we respect the patient's right to privacy, and the patient's confidential information is not compromised.

\section{Biochemical analysis}

For biochemical analysis all blood samples were collected in morning hours from the cubital vein using vacutainer technique. Serum CRP was determined by particle-enhanced immunonephelometry with the use of BN II analyzer at Department of Clinical Chemistry and Biochemistry Clinical Center University of Sarajevo. CardioPhase high-sensitivity CRP (DADE BEHRING, Marburg, Germany) was used a diagnostic reagent. CardioPhasehs-CRP consists of a suspension of polystyrene particles coated with mouse monoclonal antibodies to CRP. Reference interval for CRP with the use of this method is from 0 to $5 \mathrm{mg} / \mathrm{L}$, and the lower limit of CRP detection is $0.18 \mathrm{mg} / \mathrm{L}$. Serum NO concentration was determined at the Department of Human Physiology, Medical Faculty University of Sarajevo. Blood samples were allowed to coagulate. After coagulation the samples were centrifuged at $2000 \mathrm{~g}$ for $5 \mathrm{~min}$, and the serum samples were deproteinized by adding $750 \mu \mathrm{l}$ of distilled water and 50 $\mu 1$ of $30 \% \mathrm{ZnSO} 4$ to $250 \mu \mathrm{l}$ of each serum sample. Samples were further centrifuged at $2000 \mathrm{~g}$ for $10 \mathrm{~min}$ and frozen at $-200^{\circ} \mathrm{C}$ until the analysis was performed. 
The serum NO concentration was determined by measuring nitrite concentration, a stable metabolic product of NO with oxygen. Conversion of nitrates (NO3-) to nitrites (NO2-) was done with elementary zinc. The nitrite concentration was determined by classic colorimetrical Griess reaction (Green et al. 1982). Absorbance was measured at $546 \mathrm{~nm}$. The results were expressed as $\mu \mathrm{mol} / \mathrm{L}$.

\section{Statistical analysis}

The Shapiro-Wilk test of normality was used to test the normality and variance homogeneity of data. Data are presented as mean \pm standard error of the mean (SEM). Differences in normally distributed variables between groups were tested by ANOVA test. Coefficient of correlation was assessed by Pearson's test. A $p$ value of less then 0.05 was considered statistically significant. The software used was SPSS for Windows (version 12.0; SPSS, Chicago, IL, USA).

\section{RESULTS}

Serum NO concentration in veterans without PTSD was lower $(7.12 \pm 2.60 \mu \mathrm{mol} / \mathrm{L})$ then serum NO concentration in veterans with PTSD $(7.64 \pm 4.43 \mu \mathrm{mol} / \mathrm{L})$, but observed difference was not statistically significant. Serum NO concentration in the control group $(11.26 \pm 7.01 \mu \mathrm{mol} / \mathrm{L})$ was statistically significantly higher $(\mathrm{p}<0.05)$ compared to serum NO concentration in veterans with and without PTSD (Figure 1).

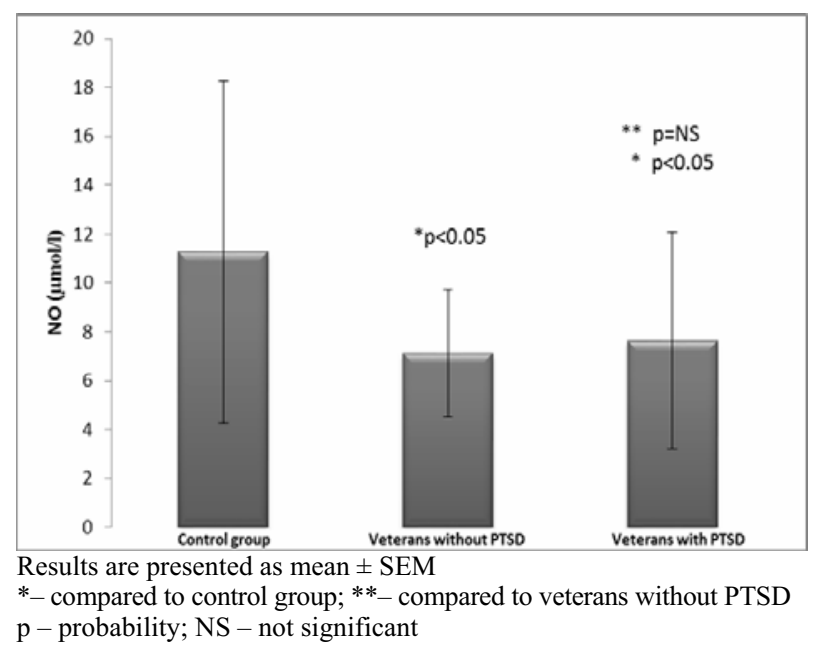

Figure 1. Serum NO concentration $(\mu \mathrm{mol} / \mathrm{L})$ in control group, veterans without PTSD and veterans with PTSD

Serum CRP concentration in veterans with PTSD $(3.54 \pm 1.19 \mathrm{mg} / \mathrm{L})$ was higher then serum CRP concentration veterans without PTSD $(3.24 \pm 2.04 \mathrm{mg} / \mathrm{L})$, but observed difference was not statistically significant $(\mathrm{p}=\mathrm{NS})$. Serum CRP concentration in veterans with and without PTSD was statistically significantly higher $(\mathrm{p}<0.05)$ compared to serum CRP concentration in control group (1.26 $\pm 1.06 \mathrm{mg} / \mathrm{L})$ (Figure 2).

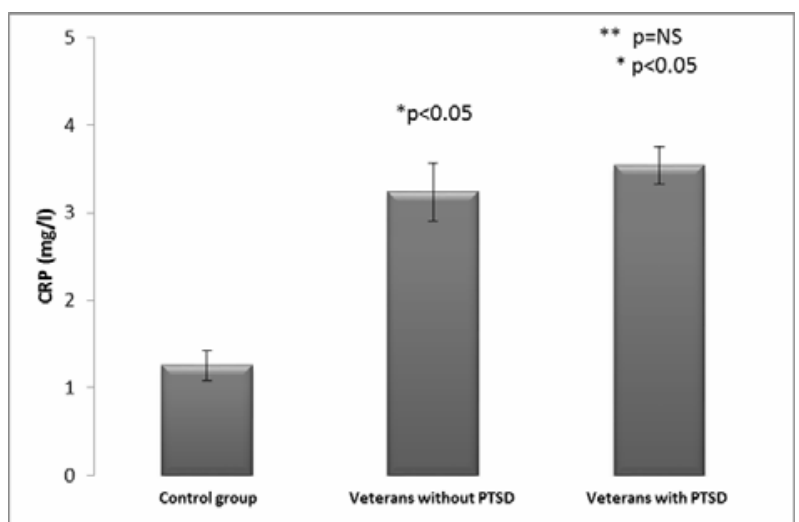

Results are presented as mean \pm SEM

*- compared to control group; **- compared to veterans without PTSD p - probability; NS - not significant

Figure 2. Serum CRP concentration $(\mathrm{mg} / \mathrm{L})$ in control group, veterans without PTSD and veterans with PTSD

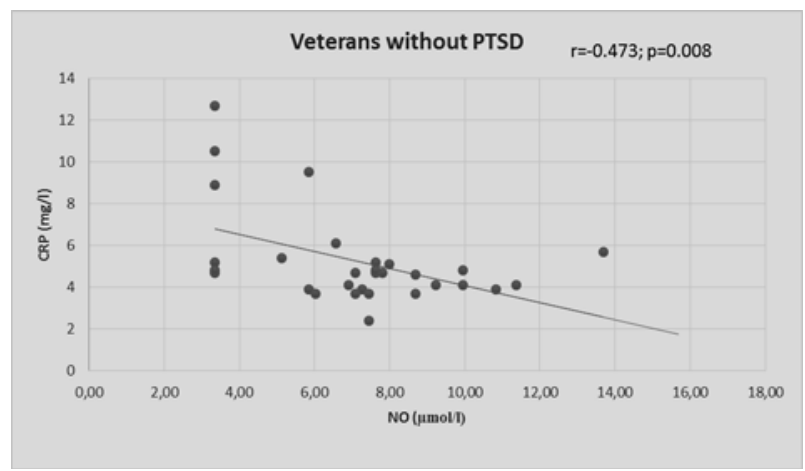

Figure 3. Correlation between serum NO and CRP concentration in veterans without PTSD

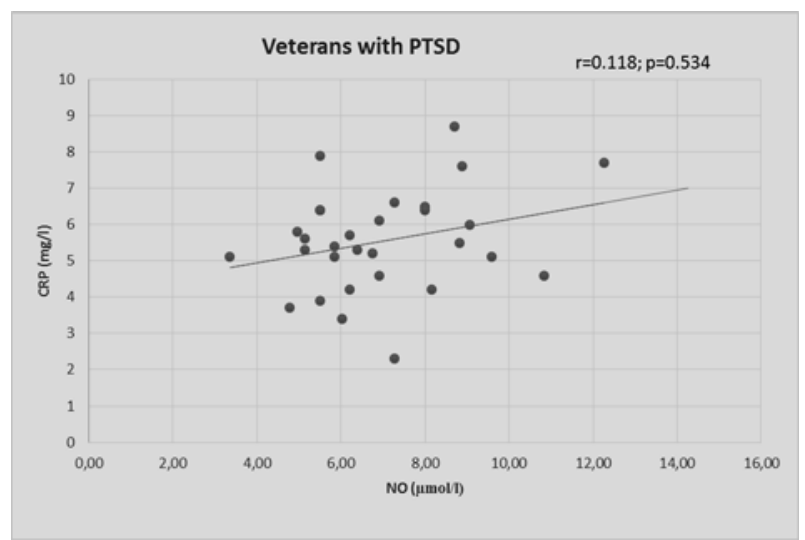

Figure 4. Correlation between serum NO and CRP concentration in veterans with PTSD

Statistically significant negative correlation was observed between serum NO and CRP concentration in veterans without PTSD $(\mathrm{r}=-0.473 ; \mathrm{p}<0.01)$ (Figure 3). No correlation was observed between serum NO and CRP concentration in veterans with PTSD $(r=0.118$; $\mathrm{p}=0.534)$ (Figure 4).

No correlation was observed between serum NO and CRP concentration in the control group of subjects ( $\mathrm{r}=$ $0.067 ; \mathrm{p}=0.727$ ) (Figure 5). 


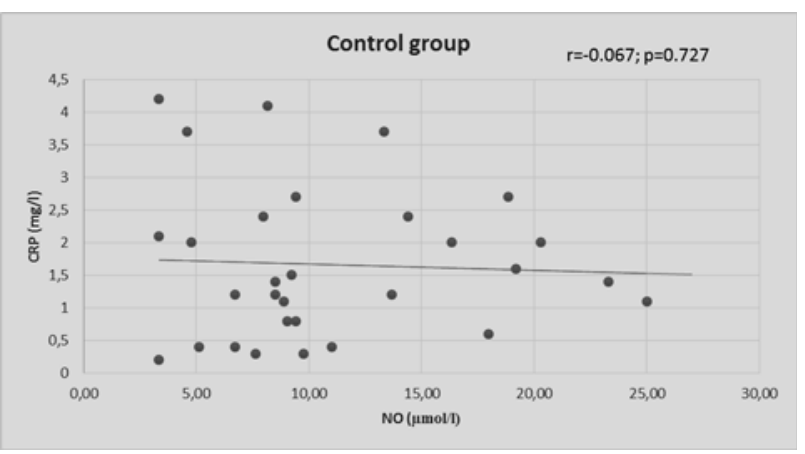

Figure 5. Correlation between serum NO and CRP concentration in the control group

\section{DISCUSSION}

To the best of our knowledge this is the first study in which serum NO and CRP concentrations were evaluated in Bosnian veterans with and without chronic PTSD. Studies so far related to NO assessment are scarce, and mainly have been conducted in conditions of acute stress. Preclinical evidence point to potential causal role of NO in anxiety-related behaviors. Because of the prominent role of NO in neuronal toxicity, cellular memory processes, and as a neuromodulator, nitrergic pathways may have an important role in stressrelated hippocampal degenerative pathology and cognitive deficits seen in patients with PTSD (Oosthuizenet al. 2005). In an accordance with this postulation, results of our study have shown significantly lower values of serum NO both in veterans with and without PTSD compared to the control group.

Yehet al. (2002) tested occurrence of acute stress and changes in serum NO concentration in 187 male individuals that were involved in rescuing actions after the earthquake in Taiwan. In 31 of them symptoms of PTSD have developed. Furthermore, negative correlation was observed between severity of stress symptoms and serum NO concentration. Pall (2001) considers that NO might have one of the pivotal role in PTSD development based on experimental findings from animal studies which have shown that damages of blood-brain barrier may be mediated by NO.

Our findings are not in accordance with data from animal models which have shown that production of NO by iNOS is increased during prolonged stress since our results have shown decreased levels of NO in veterans with and without chronic PTSD compared to control subjects without direct experience of war combat and without symptoms of PTSD. Contrary to our observation, experimental findings have demonstrated that iNOS produces in brain cells NO in a sustainable manner leading to increased levels of NO that results in local damage of neurons and in shrinking of hypocampus which is often seen in patients with PTSD (Harvey et al. 2003).
Results of our study have shown increased values of CRP in veterans with and without PTSD compared to control subjects. Veterans with PTSD had higher concentration of serum CRP compared to veterans without PTSD but difference was not statistically significant. Results obtained in our study are in accordance with results by Spitzer et al. (2010) who have reported that PTSD positive subjects had higher chances of having increased levels of CRP compared to subjects without PTSP. Authors suggested that there is a close relation between PTSD and chronic low-grade inflammation which may represent one of the psychobiological pathways from PTSD to poorer physical health and development of different diseases. Accordingly, Vaccarino et al. (2010) reported that twins with PTSD had $69 \%$ higher values of serum CRP compared to theirs twin brother without PTSD. Contrary to above findings are results by Von Känel et al. (2007) who reported increased values of TNF-alpha (TNF- $\alpha$ ) and IL-1beta (IL-1 $\beta$ ) in patients with PTSD, whereas no difference in serum CRP was observed between PTSD patients and control group.

Association between serum levels of NO and CRP in patients with and without PTSD has not been investigated extensively so far. Results of our study have determined no correlation between serum concentration of NO and CRP in the control group and in veterans with PTSD. However, statistically significant negative correlation between serum NO and CRP levels was observed in veterans without PTSD. Since by searching the literature we could not find similar study we could not compare our results with results of other authors. Possible explanation for obtained results might be proven effect of CRP on inhibition of eNOS in experimental studies (Singh et al. 2007, Hein et al. 2009). Results of these studies have shown that CRP inhibits GTP cyclohydrolase 1 and stimulates NADPH oxidase which induces decrease of tetrahydrobiopterin and increase of reactive oxygen species resulting in decreased activity of eNOS and consequently decreased bioavailability of NO. Possible explanation for unobserved correlation between CRP and NO in veterans with PTSD might be that studies thus far have shown that CRP affects different isoform of NOS in a different manner. Namely, CRP negatively influences production of eNOS, and on the other hand, CRP has shown positive influence on iNOS especially in conditions of chronic stress (Oosthuizen et al. 2005). It remains to be elucidated whether there is an imbalance between these two NOS isoforms and over stimulation of one isoform over another that contributes to insignificant correlation between CRP and NO in veterans with PTSD observed in the present study. Better understanding of potential association between serum NO and CRP is warranted is patients with PTSD.

In interpreting the findings of the current study, several limitations should be acknowledged. Firstly, the 
sample size was small consisting of veterans with and without PTSD and control group from select population and, therefore, the results cannot be generalized over the whole population. Secondly, the cross-sectional design of the study prevents us from deducing any causal relations between our findings. Thirdly, the study group consisted of patients of just one Unit for Trauma-related Disorders. However, the subjects included in the study come from different regions of the country.

Results of the present study suggest possible involvement of chronic inflammation and endothelial dysfunction in the pathogenesis of PTSD. Further large prospective population-based studies and mechanistic studies are still needed to validate our findings. Clinical relevance and importance of obtained results remain to be elucidated.

\section{CONCLUSION}

The present study has showed significant increase of serum CRP and significant decrease of serum NO concentrations in veterans with and without PTSD compared to control subjects. Furthermore, statistically significant negative correlation between serum NO and CRP concentration in veterans without PTSD was determined, where as such correlation was not observed in control subjects and veterans with PSTD. Obtained results point to complex mechanisms in PTSD pathogenesis that require further exploration.

\section{Acknowledgements: None.}

\section{Conflict of interest: None to declare.}

\section{Contribution of individual authors:}

Almir Fajkić: design of the study, recruitment and collection of data, literature searches and analyses, interpretation of data, writing of the report;

Miralem Musić: design of the study, literature searches and analyses, interpretation of data;

Alma Kulenović Džubur: design of the study, recruitment and collection of data, writing of the report;

Asija Začiragić: literature searches and analyses, interpretation of data, writing of the report;

Nesina Avdagić, Nermina Babić: statistical analyses, interpretation of data;

Orhan Lepara: design of the study, interpretation of data, writing of the report;

Esad Pepić : literature searches and analyses, writing of the report;

Amela Dervišević: literature searches and analyses, statistical analyses, interpretation of data, writing of the report;

Jasminko Huskić: design of the study, statistical analyses, interpretation of data;

\section{References}

1. Altemus M, Dhabhar FS, Yang R: Immune function in PTSD. Ann NY Acad Sci 2006; 1071:167-183.

2. Avdagić N, Zaćiragić A, Babić N, Hukić M, Seremet M, Lepara O, Nakaš-Ićindić E: Nitric oxide as a potential biomarker ininflammatory bowel disease. Bosn J Basic Med Sci 2013; 13:5-9.

3. Bloor $M$, Leyland A, Barnard $M$, McKeganey $N$ : Estimating hidden populations: a new method of calculating the prevalence of drug-injecting and noninjecting female street prostitution. Br J Addict 1991; 86:1477-83.

4. Bredt DS: Endogenous nitric oxide synthesis: biological functions and pathophysiology. Free Radic Res 1999; 31:577-596.

5. Green LC, Wagner DA, Glogowski J, Skipper PL, Wishnok JS, Tannenbaum SR: Analysis of nitrate, nitrite and $14 \mathrm{~N}$ nitrate in biological fluids. Anal Biochem 1982; 126:131-8.

6. Guix FX, Uribesalgo I, Coma M, Muñoz FJ: The physiology and pathophysiology of nitric oxide in the brain. Prog Neurobiol 2005; 76:126-52.

7. Harvey BH, Naciti C, Brand L, Stein DJ: Endocrine, cognitive and hippocampal 5-HT1A/2A receptor changes evoked by a time-dependant sensitization (TDS) stress model in rats. Brain Res 2003; 983:97-107.

8. Hein TW, Singh U, Vasquez-Vivar J, Devaraj S, Kuo L, Jialal I: Human C-reactive protein induces endothelial dysfunction and uncoupling of eNOS in vivo. Atherosclerosis 2009; 06:61-8.

9. Ibragic S, Sofic E, Suljic E, Avdagic N, Bajraktarevic A, Tahirovic I: Serum nitric oxide concentrations in patients with multiple sclerosis and patients with epilepsy. $J$ Neural Transm (Vienna) 2012; 119:7-11.

10. Lucas SM, Rothwell NJ, Gibson RM: The role of inflammation in CNS injury and disease. Br J Pharmacol 2006; 147:232-240.

11. McDade TW: Early environments and the ecology of inflammation. Proc Natl Acad Sci USA 2012; 109:17281-88.

12. Moncada S, Palmer RM, Higgs EA: Nitric oxide: physiology, pathophysiology, and pharmacology. Pharmacol Rev 1991; 43:109-42.

13. Omar SA, Webb AJ, Lundberg JO, Weitzberg E: Therapeutic effects of inorganic nitrate and nitrite in cardiovascular and metabolic diseases. J Intern Med 2016; 279:315-36.

14. Oosthuizen F, Wegener G, Harvey BH: Nitric oxide as inflammatory mediator in post-traumatic stress disorder (PTSD): evidence from an animal model. Neuropsychiatr Dis Treat 2005; 1:109-123.

15. Orida NK \& Lai ChS: Nitric oxide and renal patient. Dialysis and transplantation 2000; 29:174-185.

16. Pall ML: Common etiology of posttraumatic stress disorder, fibromyalgia, chronic fatigue syndrome and multiple chemical sensitivity via elevated nitric oxidel peroxynitrite. Medical Hypotheses 2001; 57:139-145.

17. Roberts $W$, Riba R, Homer-Vanniasinkam S, Farndale $R W$, Naseem KM:Nitric oxide specifically inhibits integrin-mediated platelet adhesion and spreading on collagen. J Thromb Haemost 2008; 6:2175-85.

18. Singh U, Devaraj S, Vasquez-Vivar J, Jialal I: C-reactive protein decreases endothelial nitric oxide synthase activity via uncoupling. J Mol Cell Cardiol 2007; 43:780-91. 
19. Spitzer C, Barnow S, Völzke H, Wallaschofski H, John U, Freyberger HJ et al.: Association of posttraumatic stress disorder with low-grade elevation of C-reactive protein: evidence from the general population. $J$ Psychiatr Res 2010; 44:15-21.

20. Vaccarino V, Bremner JD, Afzal N, Veledar E, Goldberg $J$ : Posttraumatic stress disorder is associated with higher C-reactive protein levels. J Am Coll Cardiol 2010; 55:A176.

21. Vannini $F$, Kashfi $K$, Nath $N$ : The dual role of iNOS in cancer. Redox Biol 2015; 6:334-343.

22. Von Känel R, Hepp U, Kraemer B, Traber R, Keel M, Mica L, Schnyder U: Evidence for low-grade systemic proinflammatory activity in patients with posttraumatic stress disorder. J Psychiatr Res 2007; 41:744-52.
23. Wong CM: Post-traumatic stress disorder: advances in psychoneuroimmunology. Psychiatr Clin North Am 2002; 25:369-83.

24. World Health Organization: International Statistical Classification of Diseases and Related Health Problems, 10th Revision: Instruction Manual, 2011; vol. 2.

25. Yeh CB, Leckman J, Wan FJ, Shiah IS, Lu RB: Characteristics of acute stress symptoms and nitric oxide concentration in young rescue workers in Taiwan. Psychiatry Res 2002; 112:59-68.

26. Yehuda R: Neuroendocrine aspects of PTSD. Handb Exp Pharmacol 2005; 169:371-403.

27. Zaciragic A, Mulabegovic N, Huskic J: Physiological and pathophysiological functions of C-reactive protein and its role in cardiovascular risk assessment: comprehensive review. Folia Medica 2013; 48:1-10.

Correspondence:

Almir Fajkić, MD, PhD

Department of Pathophysiology, Medical Faculty, University of Sarajevo

Čekalusa 90, 71000 Sarajevo, Bosnia and Herzegovina

E-mail:fajkic.almir@mf.unsa.ba 